\title{
Response to Comment by Ting et al.: The importance of a full-thickness vaginal wall dissection
}

\author{
${\text { Shilpa } \operatorname{Iyer}^{1,2} \text { (ID) S. M. Botros }}^{1}$
}

Received: 26 July 2017 / Accepted: 31 July 2017 /Published online: 4 September 2017

(C) The International Urogynecological Association 2017

\section{Dear Editors,}

We thank Dr Ting and his colleagues very much for their interest in our article reviewing the safety and historical trends in vaginal mesh placement for pelvic organ prolapse in their letter to the editor. In it, they propose a full-thickness dissection between the anterior vaginal wall and the bladder, as opposed to a split-thickness dissection, as a way of reducing vaginal mesh exposure rates. They describe this technique in detail. In our article, we did not describe in detail different depths of mesh dissection in relation to vaginal or bladder mesh exposure rates. In the studies we reviewed, the authors did not differentiate their mesh exposure rates by depth of dissection either.

In 2015, Dr. Noblett and her colleagues published an anatomical comparison of the location of mesh placement between abdominal sacrocolpopexy and vaginal anterior mesh placement in ten cadaveric specimens using an attempted fullthickness vaginal mesh placement. In their vaginal mesh placement, they found that they had achieved a fullthickness dissection in $50 \%$ of their patients, similar to placement of mesh for the abdominal sacrocolpopexy with one bladder injury during vaginal dissection. They conclude that a full-thickness dissection could result in lower vaginal mesh exposure rates [1]. In the extended CARE trial, the authors found the vaginal mesh exposure rate for abdominal sacrocolpopexy to be $10.5 \%$ at 7 years after surgery [2]. This study included heavier meshes, which may contribute to the higher rate of mesh exposure. Although a deeper dissection plan may reduce vaginal mesh exposure rates, we have not yet evaluated longer term data. We await the results of $\mathrm{Dr}$ Ting and colleagues and thank them again for their contribution.

\section{Compliance with ethical standards}

Conflicts of interest Shilpa Iyer and Sylvia Botros have no disclosures.

\section{References}

1. Noblett K, Brueseke T, Lin F, Rosenblatt P. Comparison of location of mesh placed transvaginally vs mesh placed abdominally at the time of sacrocolpopexy. Int Urogynecol J. 2015;26(1):79-83.

2. Nygaard I, Brubaker L, Zyczynski H, Cundiff G, Richter H, Gantz $\mathrm{M}$, et al. Long-term outcomes following abdominal sacrocolpopexy for pelvic organ prolapse. JAMA. 2013;309(19):2016-24.

This reply refers to the comment available at https://doi.org/10.1007/ s00192-017-3463-8.

Shilpa Iyer

Siyer2@bsd.uchicago.edu

1 Center for Pelvic Health, North Shore University Health Systems, Skokie, IL, USA

2 The University of Chicago, 5841 S. Maryland Avenue, MC 2050, Chicago, IL 60637, USA 